\section{EVERYTHING DENTAL FOR DECONTAMINATION}

Henry Schein Dental's experienced team of decontamination specialists has all the in-depth knowledge needed to help guide you through every aspect of the decontamination process, ensuring compliance with HTM 01-05 guidelines.

Their expert team understands how instruments should flow through the decontamination process from surgery to LDU (Local Decontamination Unit), and in conjunction with their in-house equipment specialists, can plan layouts and install equipment that makes optimal use of available space. Their outstanding array of decontamination equipment includes Melag washer disinfectors and autoclaves, which, along with infection control consumables from all the UK's leading suppliers, means that all your cleaning and sterilisation supplies can be found in one, convenient place.

Henry Schein Dental's aim is to supply 'Everything Dental' that a practice needs to be successful and profitable. An important part of this is to educate practice teams and they run several events on Decontamination and HTM 01-05. Events available include evening courses at locations nationwide, interactive CORE CPD in-practice training for your team and the new City \&t Guilds accredited course, Cross Infection and Decontamination.

For 2015 dates and venues visit www.henryschein. co.uk/education

\section{A STAND OUT MATERIAL}

Aongen Collagen Matrix stands out from other substitutes by clinical demand for its material base and scaffold design.

For a wound to regenerate healthy soft tissue it needs not only a healing period but also a product for it to implant on. Aongen Collagen Matrix is made of type I atelocollagen and GAGs which enhance healing activity at the defect site. The proper resorption profiles benefit the repair and regeneration process at remodelling stage without fibrosis or scarring. The special cross-linking property creates a better mechanical strength which maintains 3D volume stability. Aongen Collagen Matrix is the revolutionary product for tissue regeneration.

Aongen Collagen Membrane features $<50 \mu \mathrm{m}$ thickness for exceptionally flexible and adaptable advanced handling characteristics, and conforms perfectly to the surrounding bone and grafting material. The extremely dense structure provides cell occlusive properties for regenerative surgery procedures and with well-designed cross linking technology, the membrane exhibits superior tensile strength.

Aongen Collagen Membrane is made specifically to improve handing with a consistent predictable outcome. To find out more about Aongen Collagen Matrix visit www.aeonastron.com.

\title{
THE FIRST FLOWABLE RESTORATIVE MATERIAL FOR TEMPORARY TREATMENTS
}

Clip Flow is a flowable, light-curing material for all types of temporary fillings, sealings and treatments including onlay and inlay solutions. Clip Flow is also suitable as a relining material for temporary crowns and bridges, for blocking out before an impression is taken, covering the gingival margin during in-office whitening, fixing resin matrices during placement of the filling and for the temporary sealing of openings for implant screws and, during endodontic procedures, of root canal orifices.

The material in the special NDT syringe can be positioned easily and accurately and flows smoothly into the cavity, achieving good marginal adaptation. Thanks to its rapid light-curing, Clip Flow is able to achieve a sufficiently elastic consistency to enable particularly good removal of the temporary material in one piece before final placement of the filling, meaning that no additional work on the cavity is required. Clip Flow has good load-bearing capacity and is saliva-proof with tight marginal seals, thus ensuring secure temporary restorations.

For further information visit www.voco.com or email info@ voco.com.

\section{REVOLUTIONARY NEW TECHNOLOGY}

Oral-B has unveiled improvements to its App that connects to their high-performance toothbrushes by creating seamless brushing routines and allowing for expanded professional guidance.

Users can now select a personalised brushing experience to fit their oral care goals and their dental health professional can provide direct recommendations and feedback with the App. The goal for these improvements is to ensure a user can comply with their dental professionals' recommendations between visits through a streamlined App experience.

\section{Dental care journeys}

- Each personalised dental care journey is tailor-made to help users achieve their oral care goals, including whiter teeth for an upcoming wedding or fresh breath for food lovers

- The journey delivers a more active and engaged brushing routine for a pre-designed period of time, and also offers recommendations for corresponding products to match each journey

- Users can select among five journeys - fresh breath, plaque fighter, whitening, gum health or ortho care.

\section{Professional} guidance

- Dental professionals can add extra brushing time for certain focus areas in the mouth, select dental conditions and provide specific brushing and interdental cleaning instructions for their patient to follow at home

- Dental professionals can also recommend products via the App, selecting products based on their patient's specific needs

- Patients can share their compliance statistics with their hygienist and dentist via email as a way to track and discuss improvement areas and help advance their oral care routines.

For more information on Oral-B toothbrushes with Bluetooth 4.0 connectivity visit www.oralb.co.uk 